University of Nebraska - Lincoln

DigitalCommons@University of Nebraska - Lincoln

Publications, Agencies and Staff of the U.S.

Department of Commerce

U.S. Department of Commerce

2006

\title{
A bias correction for estimates of effective population size based on linkage disequilibrium at unlinked gene loci
}

Robin Waples

NOAA, robin.waples@noaa.gov

Follow this and additional works at: https://digitalcommons.unl.edu/usdeptcommercepub

Waples, Robin, "A bias correction for estimates of effective population size based on linkage disequilibrium at unlinked gene loci" (2006). Publications, Agencies and Staff of the U.S. Department of Commerce. 441.

https://digitalcommons.unl.edu/usdeptcommercepub/441

This Article is brought to you for free and open access by the U.S. Department of Commerce at DigitalCommons@University of Nebraska - Lincoln. It has been accepted for inclusion in Publications, Agencies and Staff of the U.S. Department of Commerce by an authorized administrator of DigitalCommons@University of Nebraska - Lincoln. 


\title{
A bias correction for estimates of effective population size based on linkage disequilibrium at unlinked gene loci*
}

\author{
Robin S. Waples \\ Laboratoire d'Ecologie Alpine (LECA), Génomique des Populations et Biodiversité, Université Joseph \\ Fourier, Grenoble, France (Present Address: National Marine Fisheries Service Northwest Fisheries Science \\ Center, 2725 Montlake Blvd. East, Seattle, Washington, 98112, USA, Phone: +206-860-3254; Fax: + 206- \\ 860-3335; E-mail: robin.waples@noaa.gov)
}

Received 26 August 2005; accepted 25 November 2005

Key words: computer simulations, mating systems, non-ideal populations, precision, sample size, temporal method

\begin{abstract}
Analysis of linkage disequilibrium $\left(\hat{r}^{2}=\right.$ mean squared correlation of allele frequencies at different gene loci) provides a means of estimating effective population size $\left(N_{\mathrm{e}}\right)$ from a single sample, but this method has seen much less use than the temporal method (which requires at least two samples). It is shown that for realistic numbers of loci and alleles, the linkage disequilibrium method can provide precision comparable to that of the temporal method. However, computer simulations show that estimates of $N_{\mathrm{e}}$ based on $\hat{r}^{2}$ for unlinked, diallelic gene loci are sharply biased downwards $\left(\hat{N}_{\mathrm{e}} / N<0.1\right.$ in some cases) if sample size $(S)$ is less than true $N_{\mathrm{e}}$. The bias is shown to arise from inaccuracies in published formula for $E\left(\hat{r}^{2}\right)$ when $S$ and/or $N_{\mathrm{e}}$ are small. Empirically derived modifications to $E\left(\hat{r}^{2}\right)$ for two mating systems (random mating and lifetime monogamy) effectively eliminate the bias (residual bias in $\hat{N}_{\mathrm{e}}<5 \%$ in most cases). The modified method also performs well in estimating $N_{\mathrm{e}}$ in non-ideal populations with skewed sex ratio or non-random variance in reproductive success. Recent population declines are not likely to seriously affect $\hat{N}_{\mathrm{e}}$, but if $N$ has recently increased from a bottleneck $\hat{N}_{\mathrm{e}}$ can be biased downwards for a few generations. These results should facilitate application of the disequilibrium method for estimating contemporary $N_{\mathrm{e}}$ in natural populations. However, a comprehensive assessment of performance of $\hat{r}^{2}$ with highly polymorphic markers such as microsatellites is needed.

\section{Introduction}

Although effective population size $\left(N_{\mathrm{e}}\right)$ is of central importance to both evolutionary biology and conservation biology, obtaining reliable estimates of this key parameter has proved elusive. Researchers still debate whether the ratio of effective to census population size $\left(N_{\mathrm{e}} / N\right)$ is constrained within certain limits (Nunney 1993; Frankham 1995) or can be orders of magnitude smaller for some species (Hedgecock 1994;

* The US Government's right to retain a non-exclusive, royaltyfree license in and to any copyright is acknowledged.

Turner et al. 1999; Hauser et al. 2002). Because of the difficulty of obtaining adequate demographic data to compute $N_{\mathrm{e}}$, several genetic methods for estimating effective size have been proposed. The temporal method (Krimbas and Tsakas 1971; Nei and Tajima 1981; Waples 1989), which utilizes information on the rate of change in allele frequencies between samples taken at different points in time, has been applied to a wide variety of species, and several modifications of the original approach have been proposed in recent years (Williamson and Slatkin 1999; Wang 2001; Berthier et al. 2002; Tallmon et al. 2004).
\end{abstract}


In contrast, relatively few studies (Laurie-Ahlberg and Weir 1979; Bartley et al. 1992; Bucci et al. 1997; Ardren and Kapuschinski 2003) have applied the method for estimating $N_{\mathrm{e}}$ based on gametic disequilibrium, or the non-random association of alleles at different gene loci. An advantage of this method is that it only requires a single sample from the population, as opposed to at least two for the temporal method. Hill (1981) showed that the disequilibrium method has low precision unless tightly linked loci are used, in which case the estimate is strongly affected by historical, rather than recent, $N_{\mathrm{e}}$; he concluded that the disequilibrium method has limited utility for the estimation of effective size. However, Waples (1991) pointed out that the method has greater power when effective size is small (because the signal from $N_{\mathrm{e}}$ becomes large relative to various sources of noise) and therefore may be useful for evolutionary biologists or conservation biologists, who often are concerned with low (or potentially low) $N_{\mathrm{e}}$. Waples also suggested that if data for a number of unlinked loci are available, collectively they might provide adequate precision for the method to be useful. Some empirical evaluations of the relationship between linkage disequilibrium and $N_{\mathrm{e}}$ have been made (Maruyama 1982; Hudson 1985; Vitalis and Couvet 2001), and in recent years considerable efforts have been made to analyze and model patterns of linkage disequilibrium in human populations (Hudson 2001; Pritchard and Przeworski 2001). However, most of these studies have considered large population sizes, large samples, and/or tightly linked markers, and many were motivated by goals other than estimating effective population size. No rigorous evaluation has been made of the performance of the linkage disequilibrium method for estimating contemporary $N_{\mathrm{e}}$ based on unlinked loci in natural populations.

Recently, England et al. (2006, this volume) showed that the disequilibrium method leads to severe downward bias in the estimate of $N_{\mathrm{e}}$ when sample size $(S)$ is small relative to effective size. In this paper I consider this topic in more detail, describe a method to reduce the bias to a level at which it should not impede practical application of the method, and consider precision of the resulting estimates.

\section{Methods}

Estimating linkage disequilibrium and $N_{e}$

See Table 1 for an explanation of notation. Linkage disequilibrium $(D)$ between alleles at two gene loci is defined as the difference between the observed frequency of a two-locus gamete and its expected frequency based on random association and population allele frequencies. $D$ can be estimated directly from gametic frequencies; however, for the vast majority of natural populations only genotypic data are available, which means that gamete frequencies cannot be reconstructed with certainty because of ambiguity regarding gametes that unite to form double heterozygotes. In the latter case, the most commonly used method for estimating linkage disequilibrium is Burrows' $\Delta$, which is simple to calculate and, unlike the maximum likelihood method of Hill (1974), does not depend on the assumption of random mating.

Table 1. Notation used in this paper

\begin{tabular}{|c|c|}
\hline$N$ & The number of adult individuals in a population \\
\hline$N_{\mathrm{e}}$ & Effective population size for a generation \\
\hline$k$ & $\begin{array}{l}\text { Number of gametes contributed to the } \\
\text { next generation by an individual }\end{array}$ \\
\hline $\bar{k}, V_{\mathrm{k}}$ & $\begin{array}{l}\text { Mean and variance of } k \text { among individuals } \\
\text { within a generation }\end{array}$ \\
\hline$S$ & $\begin{array}{l}\text { The number of individuals sampled to } \\
\text { estimate population parameters }\end{array}$ \\
\hline$L$ & The number of diallelic loci examined \\
\hline$J$ & $\begin{array}{l}\text { The number of pairwise comparisons of } \\
L \text { loci }[J=L(L-1) / 2]\end{array}$ \\
\hline$c$ & $\begin{array}{l}\text { Frequency of recombination between } \\
\text { two gene loci }\end{array}$ \\
\hline$\Delta$ & Burrows' composite disequilibrium measure \\
\hline$r_{\Delta}$ & $\begin{array}{l}\text { The inter-locus correlation of allele frequencies } \\
\text { based on Burrows' } \Delta\end{array}$ \\
\hline$\hat{r}^{2}$ & $\begin{array}{l}\text { The mean of squared } \hat{r}_{\Delta} \text { values } \\
\text { averaged over } J \text { pairs of loci in a single replicate }\end{array}$ \\
\hline$\overline{\hat{r}}^{2}$ & The mean of $\hat{r}^{2}$ values across all replicates \\
\hline $\begin{array}{l}E\left(\hat{r}^{2}\right), \\
V\left(\hat{r}^{2}\right)\end{array}$ & The expected value (and variance) of $\hat{r}^{2}$ \\
\hline$E\left(\hat{r}_{\mathrm{drift}}^{2}\right)$ & $\begin{array}{l}\text { Expected contribution to } \hat{r}^{2} \text { from a finite } \\
\text { number of breeders }\end{array}$ \\
\hline$E\left(\hat{r}_{\text {sample }}^{2}\right)$ & $\begin{array}{l}\text { Expected contribution to } \hat{r}^{2} \text { from sampling } \\
\text { a finite number of individuals }\end{array}$ \\
\hline$\hat{r}^{2 \prime}$ & $\begin{array}{l}\text { Empirical } \hat{r}^{2} \text { adjusted for sampling error: } \\
\hat{r}^{2 \prime}=\hat{r}^{2}-E\left(\hat{r}_{\text {sample }}^{2}\right)\end{array}$ \\
\hline$\phi$ & $\begin{array}{l}V\left(\hat{r}^{2}\right) /\left(\overline{\hat{r}}^{2}\right)^{2}=\text { squared coefficient } \\
\text { of variation of } \hat{r}^{2}\end{array}$ \\
\hline
\end{tabular}


Weir (1979) evaluated both Hill's and Burrows' methods and concluded that $\Delta$ should generally be preferred even when it is reasonable to assume random mating. An unbiased estimate of $\Delta$ can be obtained by adjusting for sample size (Weir 1979):

$$
\hat{\Delta}=\Delta \frac{S}{S-1} .
$$

$D$ values can be standardized to adjust for the effect of allele frequencies, yielding a correlation coefficient $(r)$. An estimate of the analogue to $r$ for the Burrows method $\left(r_{\Delta}\right)$ is

$$
\hat{r}_{\Delta}=\frac{\hat{\Delta}}{\sqrt{\left[\hat{p}(1-\hat{p})+\left(h_{1}-\hat{p}^{2}\right)\right]\left[\hat{q}(1-\hat{q})+\left(h_{2}-\hat{q}^{2}\right)\right]}},
$$

where $h_{1}\left(h_{2}\right)$ is the observed frequency of AA (BB) homozygotes at the first (second) locus and $\hat{p}(\hat{q})$ is the sample frequency of allele A (B) (Weir 1996). The analyses described here follow Weir and many others and use the Burrows method to compute $\hat{r}$.

In a stable population with random mating and no selection, migration, or mutation, $E(\Delta)=E\left(r_{\Delta}\right)=0$ for unlinked loci. However, in finite populations $|\Delta|$ and $\left|r_{\Delta}\right|$ will generally not be zero because of random deviations from expected probabilities. Sampling also introduces disequilibria, and the magnitude of these random departures from equilibrium will increase as $N_{\mathrm{e}}$ and $S$ decrease. Thus, it is expected that the squared coefficients of disequilibrium $\hat{\Delta}^{2}$ and $\hat{r}_{\Delta}^{2}$ will be inversely proportional to $N_{\mathrm{e}}$ and $S$. Although $E\left(\hat{r}^{2}\right)$ is not known, most authors (e.g., Hill and Robertson 1968; Ohta and Kimura 1969; Sved and Feldman 1973; Weir and Hill 1980) have used the following approach to obtain an approximation: $E\left(\hat{r}^{2}\right)=E^{2}(\hat{r})+V(\hat{r})$; since $E^{2}(\hat{r})=0$, this leads to

$$
\begin{aligned}
E\left(\hat{r}^{2}\right) & =V(\hat{r})=V\left(\frac{\hat{D}}{\sqrt{p(1-p) q(1-q)}}\right) \\
& \approx \frac{E\left(\hat{D}^{2}\right)}{E[p(1-p) q(1-q)]}, \text { and } \\
E\left(\hat{r}_{\Delta}^{2}\right) & \approx \frac{E\left(\hat{\Delta}^{2}\right)}{E\left(\left[\hat{p}(1-\hat{p})+\left(h_{1}-\hat{p}^{2}\right)\right]\left[\hat{q}(1-\hat{q})+\left(h_{2}-\hat{q}^{2}\right)\right]\right)} .
\end{aligned}
$$

Weir and Hill (1980) showed that the expectation of squared disequilibrium coefficients is strongly affected by the mating system and recombination fraction (c) between the loci in question. Assuming $N_{\mathrm{e}}$ and $S$ are both large, for monoecious species or dioecious species with random mating and no permanent pair bonds,

$$
E\left(\hat{r}_{\Delta}^{2}\right) \approx \frac{(1-c)^{2}+c^{2}}{2 N_{\mathrm{e}} c(2-c)}+\frac{1}{S},
$$

(Weir and Hill 1980), whereas with lifetime monogamy,

$$
E\left(\hat{r}_{\Delta}^{2}\right) \approx \frac{1-c+2 c^{2}}{2 N_{\mathrm{e}} c(2-c)}+\frac{1}{S} .
$$

If the loci are unlinked $(c=0.5)$, these equations simplify to

$$
\begin{aligned}
E\left(\hat{r}_{\Delta}^{2}\right) \approx & \frac{1}{3 N_{\mathrm{e}}}+\frac{1}{S} \text { (monoecious or } \\
& \text { dioecious random mating }, \\
E\left(\hat{r}_{\Delta}^{2}\right) \approx & \frac{2}{3 N_{\mathrm{e}}}+\frac{1}{S} \text { (dioecious, } \\
& \text { lifetime monogamy) }
\end{aligned}
$$

[Waples (1991) indicated that the first term in Equation (6) was $5 /\left(12 N_{\mathrm{e}}\right)$, but that is correct only for the case of gametic data.] Hereafter, I will drop the subscript and use $\hat{r}^{2}$ to refer to $\hat{r}_{\Delta}^{2}$. In both equations, $E\left(\hat{r}^{2}\right)$ can be expressed as the sum of a term due to finite population size and a term due to sampling a finite number of individuals: $E\left(\hat{r}^{2}\right)=E\left(\hat{r}_{\text {drift }}^{2}\right)+E\left(\hat{r}_{\text {sample }}^{2}\right)$. Equations (5) and (6) indicate that $E\left(\hat{r}_{\text {sample }}^{2}\right)=1 / S$ is the same in the two mating systems, but $E\left(\hat{r}_{\text {drift }}^{2}\right)$ is twice as large if permanent pair bonds are formed. Replacing $E\left(\hat{r}^{2}\right)$ with its estimate $\left(\hat{r}^{2}\right)$ and rearranging leads to an estimator for $N_{\mathrm{e}}$ :

$$
\begin{aligned}
\hat{N}_{\mathrm{e}}= & \frac{1}{3\left(\hat{r}^{2}-1 / S\right)} \text { (monoecious or } \\
& \text { dioecious random mating), } \\
\hat{N}_{\mathrm{e}}= & \frac{2}{3\left(\hat{r}^{2}-1 / S\right)}(\text { dioecious, } \\
& \text { lifetime monogamy }) .
\end{aligned}
$$

If $S$ varies among loci, the harmonic mean should be used. 


\section{Performance of the estimators}

Computer simulations were used to model the two mating systems described above in a single population closed to migration. Generations were discrete, population size $(N)$ was constant, and the numbers of males and females were equal. In the random mating model, each progeny was produced by mating a randomly selected female and a randomly selected male, chosen independently and with replacement from the breeding population. In the monogamy model, the only difference was that each progeny was produced by randomly drawing, with replacement, a permanently bonded malefemale pair. Selfing was not permitted in either model. Under the simulated conditions, the distribution of reproductive success is binomial and inbreeding effective size with or without monogamy is given by (Wright 1969, as modified by Balloux 2004):

$$
N_{\mathrm{e}}=N+1 /(2 N)+0.5 .
$$

The 0.5 term in Equation (9) (and in Equations (10) and (11) below) arises because by convention the "ideal" population is defined to include random selfing, and inbreeding is retarded slightly if selfing is not allowed. Equation (9) was used to calculate the true $N_{\mathrm{e}}$ for each simulation. For simplicity, although the last two terms on the right side of Equation (9) were carried through in all calculations and analyses reported here, in the text the true $N_{\mathrm{e}}$ is reported as the number of "ideal" individuals $(N)$.

Each replicate was initialized by choosing genotypes in generation 0 by binomial draw based on parametric allele frequencies $\left(P_{0}\right)$ at $L$ diallelic and independent gene loci. This is equivalent to drawing the initial generation of breeders from an infinitely large source population in which linkage disequilibrium is zero. Under this scenario, $r^{2}$ will increase to its asymptotic value at a rate described by Sved (1971), and during that time period estimates of $N_{\mathrm{e}}$ will be upwardly biased because $r^{2}$ is lower than its equilibrium value. Previous results for the same simulation model used here (Waples 2005) showed that, as expected for unlinked loci, $\hat{r}^{2}$ approaches its asymptotic value quickly at the rate predicted by Sved (1971), and $\hat{N}_{\mathrm{e}}$ stabilized within 3-4 generations. Therefore, the population was allowed to equilibrate for four generations (generations 0-3), after which data were collected for six generations (generations 4-9) before the simulation was terminated and another replicate begun. Limiting each replicate to 9 generations minimized the number of alleles that became extinct or drifted to extreme frequencies. To further reduce possible biases due to low frequency alleles, if the breeders (or the sample) in any generation had an allele frequency at any locus less than a threshold value $P^{*}$ (or greater than $\left.1-P^{*}\right)$, the breeders or sample were redrawn. $P^{*}=0.05$ was used in most simulations, although other values were evaluated for their effects on the results.

The relationship between $\hat{r}^{2}, S$, and true $N_{\mathrm{e}}$ was examined in a complete matrix of $N_{\mathrm{e}} \times S$ values, using $N_{\mathrm{e}}=10,12,16,20,24,30,40,60,80,100$, $120,160,200,300,500 ; S=10,12,16,20,24,30$, $40,50,60,80,100,150$, and 200 . In addition, sampling from a population with $N_{\mathrm{e}}=\infty$ was mimicked by using results for samples drawn in generation 0. Samples were taken by producing additional progeny beyond those needed for the next generation; hence, $S$ could exceed $N_{\mathrm{e}}$ (as can occur if $N_{\mathrm{e}} / N<1$ or if a species is sampled as juveniles). In each sample in each generation, $\hat{r}_{\Delta}$ was computed for each pair of loci using Equation (2), and these squared $\hat{r}_{\Delta}$ values $\left(\hat{r}^{2}\right)$ were averaged across all pairs of loci to obtain the estimate of $r^{2}$ for that sample. To obtain an overall estimate of $r^{2}$ for each parameter set, the mean $\hat{r}^{2}$ values were averaged across generations 4-9 within each replicate and then across replicates, yielding an overall mean $\overline{\hat{r}}^{2}$. Typically, 30,000 replicates were run for each parameter set, so each mean $\overline{\hat{r}}^{2}$ value (and associated $\hat{N}_{\mathrm{e}}$ values) is based on data for $6 \times 30,000=180,000$ replicate samples from a single generation.

\section{Non-ideal populations}

For a limited number of parameter sets, I also considered populations that departed from ideal conditions in either of two ways. If sex ratio is uneven but the distribution of reproductive success is random within each sex, effective size is given by

$$
N_{e} \approx \frac{4 N_{\mathrm{m}} N_{\mathrm{f}}}{N_{\mathrm{m}}+N_{\mathrm{f}}}+0.5,
$$

where $N_{\mathrm{m}}$ and $N_{\mathrm{f}}$ are the numbers of the two sexes. Uneven sex ratio was modeled by arbitrarily 
dividing the $N$ individuals each generation into two sexes in fixed proportions ranging from 1:1 to 1:15, which, based on Equation (10) and using $N=96$ or 192 , led to expected $N_{\mathrm{e}} / N$ ratios of 0.24 to 1.0 .

More generally, departures from ideal conditions can be described in terms of the mean $(\bar{k})$ and variance $\left(V_{\mathrm{k}}\right)$ among individuals in the number of gametes contributed to the next generation:

$$
N_{\mathrm{e}}=\frac{\bar{k} N-2}{\bar{k}-1+V_{\mathrm{k}} / \bar{k}}+0.5 \text {. }
$$

Equation (11) gives the inbreeding effective size for species with separate sexes and for non-selfing monoecious species (Crow and Denniston 1988; Caballero 1994).

The ratio $V_{\mathrm{k}} / \bar{k}$, termed the "index of variability" by Crow and Morton (1955), quantifies departures from ideal conditions. In the WrightFisher model, variance in reproductive success is binomial ( $=$ Poisson in the limiting case of infinite $N)$, so $V_{\mathrm{k}} \approx \bar{k}$ and $V_{\mathrm{k}} / \bar{k} \approx 1$. Here, the interest is in modeling an overdispersed Poisson distribution with mean $\bar{k}=2$ (so the population is constant in size) and variance $V_{\mathrm{k}} \geq 2$. This can be obtained via a two-step process. First, a random variable $\gamma$ is generated from an appropriate Gamma distribution (see Anderson 2001 for a similar method using the negative binomial distribution). Second, $\gamma$ is used as the parameter for a Poisson distribution, from which another number $(k)$ is randomly chosen; the value of $k$ is the number of gametes one individual contributes to the next generation. This two-step process is repeated $N$ times to generate the distribution of reproductive success values for the population as a whole. It can be shown that if the desired mean is $\bar{k}=2$ and the desired variance is $V_{\mathrm{k}}$, then the appropriate Gamma distribution is one with $\bar{\gamma}=1$ and $V_{\gamma}=\left(V_{\mathrm{k}}-2\right) / 4$. With $\bar{k}$ fixed at 2, I modeled selected values of $V_{\mathrm{k}}$ between 2 and 20 , leading to $V_{\mathrm{k}} / \bar{k}$ ratios of 1 to 10 and expected $N_{\mathrm{e}} / N$ ratios ranging from 0.19 to 1.0 . Because this procedure only leads asymptotically to $\bar{k}=2$ for large samples, in each generation I adjusted the actual mean reproductive success to $\bar{k}=2$ by adding or subtracting progeny from randomly chosen individuals. Realized $V_{\mathrm{k}}$ was calculated after the adjustment, averaged across replicates, and used in Equation (11) to calculate true $N_{\mathrm{e}}$ for each parameter set.
To validate general behavior of the simulation model under non-ideal conditions, I also tracked temporal changes in allele frequency and estimated $N_{\mathrm{e}}$ using the temporal method. Allele frequencies were computed for Plan I samples (Nei and Tajima 1981; Waples 1989) taken in generation 3, and these were compared with frequencies in samples from generations 4-9 using Pollak's (1983) method to estimate the standardized variance in allele frequencies $(F)$. The estimates of $F$ for 1-6 elapsed generations were used to estimate $N_{\mathrm{e}}$ using Equation (12) in Waples (1989), and the harmonic mean of the $\hat{N}_{\mathrm{e}}$ values for the different time periods was taken as the temporal estimate of $N_{\mathrm{e}}$ for that parameter set.

\section{Results}

\section{Evaluating bias}

Random mating

Mean $\hat{r}^{2}$ and $\overline{\hat{r}}^{2}$ values did not depend on the number of loci used, nor on the initial allele frequency in the range $P_{0}=0.5-0.9$ (data not shown). Therefore, except as noted below, results will be shown only for $L=8$ and $P_{0}=0.5$.

It is apparent that Equation (7) does not provide an unbiased estimate of effective population size for the range of $S / N_{\mathrm{e}}$ ratios likely to occur in the study of natural populations (Figure 1). $\hat{N}_{\mathrm{e}}$ is downwardly biased if $S$ is less than about $2 N_{\mathrm{e}}$, and the bias is substantial for $S<N_{\mathrm{e}}$, particularly for samples of small absolute size. If $S / N_{\mathrm{e}}<0.1, \hat{N}_{\mathrm{e}}$ can be a small fraction of true $N_{\mathrm{e}}$. When $S$ is small relative to $N_{\mathrm{e}}, \hat{N}_{\mathrm{e}}$ is more closely related to the sample size than it is to the true effective size. Conversely, for $S>2 N_{\mathrm{e}}$, Equation (7) leads to an overestimate of $N_{\mathrm{e}}$, although the bias is less pronounced $(<20 \%$; Figure 1). Again, for the same $S / N_{\mathrm{e}}$ the upward bias in $\hat{N}_{\mathrm{e}}$ is larger for smaller samples.

Because the $S /(S-1)$ correction (Equation (1)) to obtain an unbiased estimate of $\Delta$ increases $\hat{r}^{2}$, it exacerbates the downward bias in $\hat{N}_{\mathrm{e}}$ for small $S$. Therefore, behavior of $\hat{r}^{2}$ and $\hat{N}_{\mathrm{e}}$ was also evaluated without this adjustment. Omitting the $S /(S-1)$ term does reduce downward bias (but only moderately) for small $S / N_{\mathrm{e}}$, but it increases upward bias for large $S / N_{\mathrm{e}}$ (data not shown). As 




Figure 1. Random mating model. The ratio of estimated to true effective size $\left(\hat{N}_{\mathrm{e}} / N_{\mathrm{e}}\right)$ for simulated data with $S=10-200$ and $N_{\mathrm{e}}=10-500$. Mean $\overline{\hat{r}}^{2}$ values were computed as in Equation (2) and used to compute $N_{\mathrm{e}}$ in Equation (7). Vertical dashed line indicates that the region of no bias $\left(\hat{N}_{\mathrm{e}}=N_{\mathrm{e}}\right)$ occurs at $S \approx 2.15 N_{\mathrm{e}}$.

the $S /(S-1)$ adjustment to $\Delta$ is implemented in widely used computer programs for analyzing population genetic data (e.g., LINKDOS: Black and Krafsur 1985; Garnier-Gere and Dillmann 1992; GENEPOP: Raymond and Rousset 1995), and omitting it does not solve the bias problem, the adjustment was used in the remaining analyses. Because Equations (3)-(6) were derived by approximating $E\left(\hat{r}^{2}\right)$ as the ratio of two expectations, I also evaluated performance of a modified $\hat{r}^{2}$ based on computing an average (across all pairs of loci) of the squared numerator of Equation (2) divided by an average (across all pairs of loci) of the squared denominator of Equation (2). Vitalis and Couvet (2001) used this approach - averaging numerators and denominators separately - with their index of multilocus gametic disequilibrium. For all parameter sets examined, however, this approach led to a larger $\hat{r}^{2}$, which increased bias in $\hat{N}_{\mathrm{e}}$ for small samples; therefore, this method was not considered further.

\section{Monogamy}

Results for the monogamy model (not shown) were qualitatively similar: substantial downward bias for $S / N_{\mathrm{e}}<1$ and upward bias for $S / N_{\mathrm{e}}>1$. The downward bias for small $S / N_{\mathrm{e}}$ was not quite as pronounced as in the random mating model, which is understandable because $E\left(\hat{r}_{\text {drift }}^{2}\right)$ is larger with monogamy, so the contribution from sampling is relatively less important. Conversely, upward bias for large $S / N_{\mathrm{e}}$ was more severe in the monogamy model (up to $30 \%$ in some cases for $S / N_{\mathrm{e}}>5$ ).

For both mating systems, the downward (upward) bias in $\hat{N}_{\mathrm{e}}$ occurs when $\hat{r}^{2}$ is larger (smaller) than the value expected from Equations (5) and (6) based on the true $N_{\mathrm{e}}$. I evaluated two different approaches to reduce this bias, one based on modifying $E\left(\hat{r}^{2}\right)$ to more closely predict empirical $\hat{r}^{2}$, the other based on adjusting $\hat{r}^{2}$ before using Equation (7) to calculate $\hat{N}_{\mathrm{e}}$.

\section{Empirical modification to $E\left(\hat{r}^{2}\right)$}

\section{Random mating}

To obtain the approximations for $E\left(\hat{r}^{2}\right)$ given in Equations (3)-(6), Weir and Hill (1980) assumed both $S$ and $N_{\mathrm{e}}$ were small enough that second order and higher terms, as well as inverse terms in $S N_{\mathrm{e}}$, could be ignored. Accordingly, I evaluated empirically whether inclusion of second order terms could be effective in removing the bias in $\hat{N}_{\mathrm{e}}$. To isolate the contribution to $\hat{r}^{2}$ of sampling from that due to drift, I first examined data for samples taken only in generation 0 , which mimics sampling from a population of 
infinite size. In this case, $E\left(\hat{r}_{\text {drift }}^{2}\right)=0$, so observed $\overline{\hat{r}}^{2}=\overline{\hat{r}}_{\text {sample }}^{2}$, which could be compared with its expectation from Equation (5) $(1 / S)$. In all cases, empirical $\overline{\hat{r}}^{2}$ was larger than $1 / S$, and residuals were larger for small $S$ (Figure 2a). A best-fit model of the form $y=a / S^{2}+b / S+c$ yielded $c=4.8 \times 10^{-5}$ and $b=0.991$, in good agreement with the theoretical expectation that $c=0$ and $b=1$ (Equation (5)). Setting $c=0$ and $b=1$, the least-squares fit $\left(r^{2}>0.999\right)$ to the empirical data is residual $\overline{\hat{r}}_{\text {sample }}^{2}=3.19 / S^{2}$. Thus, Equation (5) can be improved by adding a second order term for sampling error: $E\left(\hat{r}_{\text {sample }}^{2}\right) \approx 1 / S+3.19 / S^{2}$.
Evaluations indicated that this adjustment is less effective for $S<30$, so an adjusted $E\left(\hat{r}_{\text {sample }}^{2}\right)$ was computed separately for $S=10-30$. In this case, a better fit $\left(r^{2}>0.999\right)$ to the empirical data was found by allowing $c$ and $b$ to depart slightly from values in Equation (5): $E\left(\hat{r}_{\text {sample }}^{2}\right) \approx 0.0018+0.907 / S+4.44 / S^{2}$ (Figure $2 b$ ).

To evaluate effectiveness of these modifications to $E\left(\hat{r}_{\text {sample }}^{2}\right)$ with finite $N_{\mathrm{e}}$, I subtracted the modified $E\left(\hat{r}_{\text {sample }}^{2}\right)$ from empirical $\overline{\hat{r}}^{2}$ values, leaving a quantity $\hat{r}^{2 \prime}=\hat{r}^{2}-E\left(\hat{r}_{\text {sample }}^{2}\right)$ that can be compared with the expected contribution from drift $\left(E\left(\hat{r}_{\text {drift }}^{2}\right)\right)$. For each $N_{\mathrm{e}}, \hat{r}^{2 \prime}$ was aver-
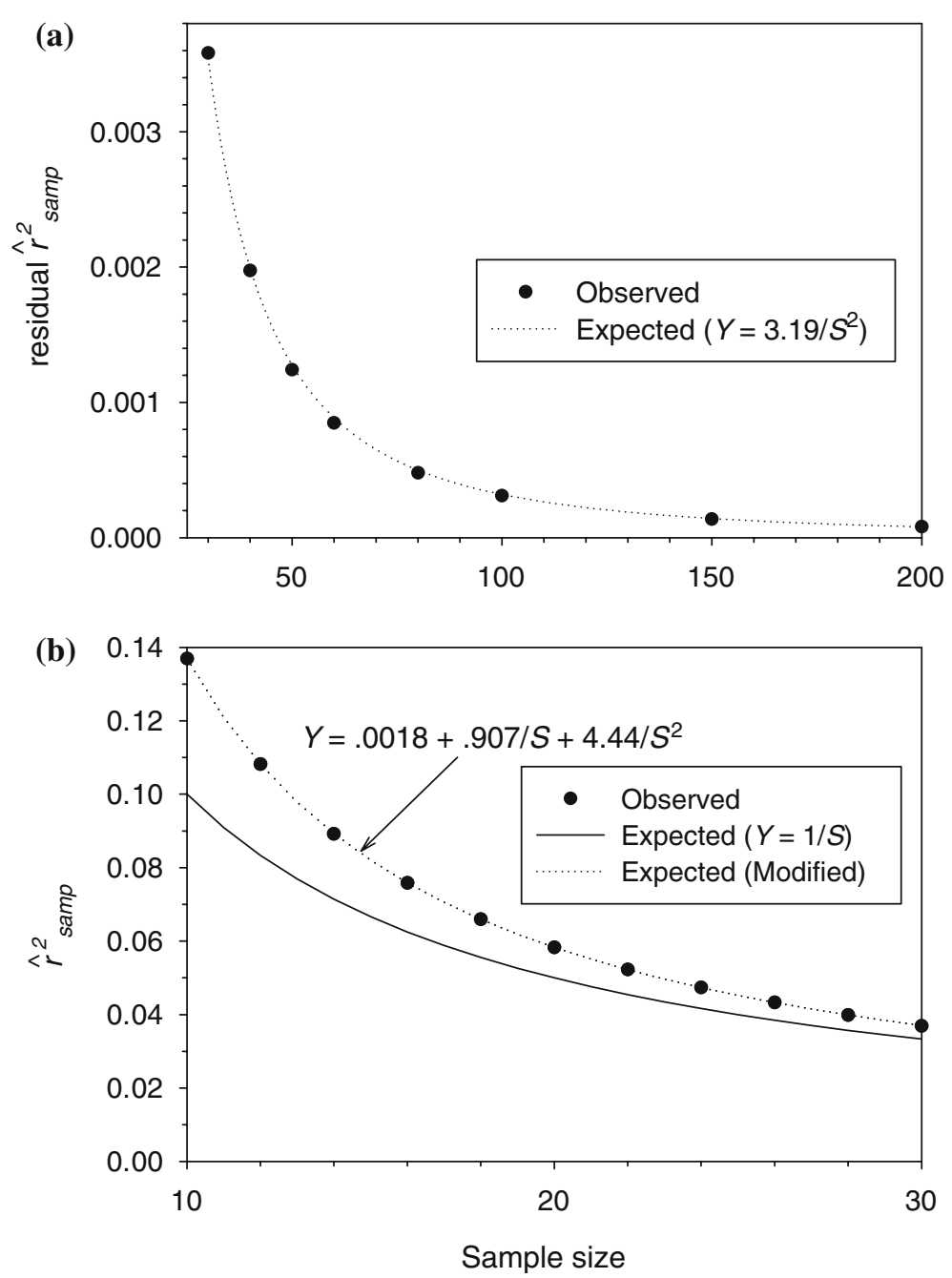

Figure 2. Random mating model. A. Large sample sizes $(S \geq 30)$. Residual $\overline{\hat{r}}^{2}$ after subtracting the expected contribution from sampling $\left(E\left(\overline{\hat{r}}_{\text {samp }}^{2}\right)=1 / S\right.$; Equation (5)) for simulations that mimic sampling for a population with $N_{\mathrm{e}}=\infty$. Filled circles are empirical results from simulations; dotted line is the curve $Y=3.19 / S^{2}$ fitted to the residuals. B. Small sample sizes $(S \leq 30)$. Filled circles are empirical $\overline{\hat{r}}^{2}$ for simulations that mimic sampling for a population with $N_{\mathrm{e}}=\infty$; solid line is $E\left(\overline{\hat{r}}_{\text {samp }}^{2}\right)=(1 / S)$ and dotted line is the modified $E\left(\hat{\bar{r}}_{\text {samp }}^{2}\right)=0.0018+0.907 / S+4.44 / S^{2}$ fitted to the data. 
aged across sample sizes, separately for $S \geq 30$ and $S<30$. If the drift term in Equation (5) were adequate, these mean $\hat{r}^{21}$ values should closely approximate $1 /\left(3 N_{e}\right)$. Although agreement was reasonably good for $N_{\mathrm{e}}$ larger than about 100 , for smaller effective sizes the residuals $\left[\hat{r}^{2 \prime}-1 /\left(3 N_{\mathrm{e}}\right)\right]$ were substantial and negative (Figure 3$)$, indicating that $1 /\left(3 N_{\mathrm{e}}\right)$ slightly overestimates the contribution of drift to $\overline{\hat{r}}^{2}$. For $S \geq 30$, the least-squares fit yielded $c<2 \times 10^{-5}$ and $b=0.325$, again in good agreement with the theoretical expectation $(c=0, b=1 / 3$; Equation 5). Setting $c=0$ and $b=1 / 3$, the least-squares fit to $\hat{r}^{2 \prime}$ occurred with a second order-term of $-0.69 / N_{\mathrm{e}}^{2}$ (Figure 3a). For $S<30$, a better fit was found for $E\left(\hat{r}_{\text {drift }}^{2}\right) \approx 0.308 / N_{\mathrm{e}}-0.52 / N_{\mathrm{e}}^{2}$ (Figure 3b).

Collectively, these results suggest that Equation (5) can be modified as follows to provide a better approximation for drift and sampling terms in $E\left(\hat{r}^{2}\right)$ :

$$
\begin{aligned}
E\left(\hat{r}^{2}\right)_{(S \geq 30)} & \approx\left[\frac{1}{3 N_{\mathrm{e}}}-\frac{0.69}{N_{\mathrm{e}}^{2}}\right] \mathrm{drift} \\
& +\left[\frac{1}{S}+\frac{3.19}{S^{2}}\right] \text { sample. } \\
E\left(\hat{r}^{2}\right)_{(S<30)} & \approx\left[\frac{0.308}{N_{\mathrm{e}}}-\frac{0.52}{N_{\mathrm{e}}^{2}}\right] \mathrm{drift} \\
& +\left[0.0018+\frac{0.907}{S}+\frac{4.44}{S^{2}}\right] \text { sample }
\end{aligned}
$$



Figure 3. Random mating model. A. Large sample sizes $(S \geq 30)$. Residual $\overline{\hat{r}}_{\text {drift }}^{2}$ after subtracting the expected contribution from sampling $\left[E\left(\overline{\hat{r}}_{\mathrm{samp}}^{2}\right)=1 / S+3.19 / S^{2}\right.$; Table 2] and the drift term from Equation (5) $\left(1 / 3 N_{\mathrm{e}}\right)$. Filled circles are empirical results from simulations; dotted line is the curve $Y=-0.69 / N_{\mathrm{e}}^{2}$ fitted to the residuals. B. Small sample sizes $(S \leq 30)$. Open triangles are residual $\overline{\hat{r}}_{\text {drift }}^{2}$ after subtracting $E\left(\overline{\hat{r}}_{\text {samp }}^{2}\right)=0.0018+0.907 / S+4.44 / S^{2}$ and the drift term from Equation $(5)\left(1 / 3 N_{\mathrm{e}}\right)$. Filled triangles are residuals after adjusting the drift term to $E\left(\overline{\hat{r}}_{\text {drift }}^{2}\right)=0.308 / N_{\mathrm{e}}-0.52 / N_{\mathrm{e}}^{2}$. 
Because $E\left(\hat{r}_{\text {drift }}^{2}\right)$ involves second-order terms in $N_{\mathrm{e}}$, this does not lead to as simple an expression for $\hat{N}_{\mathrm{e}}$ as does Equation (7). However, $E\left(\hat{r}_{\text {sample }}^{2}\right)$ and $\hat{r}^{2}$ can both be calculated directly from empirical data, and a solution for $\hat{N}_{\mathrm{e}}$ can be found by rearranging terms and use of the quadratic formula. For example, for $S \geq 30$,

$$
\begin{aligned}
\hat{r}^{2 \prime}= & \hat{r}^{2}-E\left(\hat{r}_{\text {sample }}^{2}\right) \\
= & \hat{r}^{2}-\left[\frac{1}{S}+\frac{3.19}{S^{2}}\right] \approx \frac{1}{3 N_{\mathrm{e}}}-\frac{.69}{N_{\mathrm{e}}^{2}} ; \\
\hat{r}^{2 \prime}= & \frac{(1 / 3) N_{\mathrm{e}}-.69}{N_{\mathrm{e}}^{2}} ; \\
& \hat{r}^{\prime \prime} N_{\mathrm{e}}^{2}-(1 / 3) N_{\mathrm{e}}+.69=0 ; \\
\hat{N}_{\mathrm{e}}= & \frac{1 / 3+\sqrt{1 / 9-2.76 \hat{r}^{2 \prime}}}{2 \hat{r}^{2 \prime}} .
\end{aligned}
$$

Expressions for $E\left(\hat{r}_{\text {sample }}^{2}\right), E\left(\hat{r}_{\text {drift }}^{2}\right)$, and $\hat{N}_{\mathrm{e}}$ for large and small sample sizes are shown in Table 2. If $\hat{r}^{\prime \prime}<0$, then all the disequilibrium can be explained by sampling error and the appropriate estimate of $N_{\mathrm{e}}$ is $\infty$ (Laurie-Ahlberg and Weir 1979). Conversely, if $\hat{r}^{2 \prime}$ is large, the square-root term can be negative; this implies small $N_{\mathrm{e}}$, and an approximate estimate is $\hat{N}_{\mathrm{e}}=(1 / 3) /\left(2 \hat{r}^{2 \prime}\right)$ for random mating or $\hat{N}_{\mathrm{e}}=(2 / 3) /\left(2 \hat{r}^{2 \prime}\right)$ for monogamy.

Use of the formulas in Table 2 to estimate $N_{\mathrm{e}}$ based on mean $\overline{\hat{r}}^{2}$ values from a new round of simulations led to results shown in Figure 4. For $S \geq 30$, bias in $\hat{N}_{\mathrm{e}}$ was virtually eliminated (less than 5\%), even for the minimum $S=30$ and $S / N_{\mathrm{e}}=0.06$ (Figure 4a). For smaller sample sizes, performance was nearly as good; bias was $<10 \%$ for all parameter sets with $S / N_{\mathrm{e}}>0.1$ and no larger than $15 \%$ for smaller samples (Figure $4 b$ ).
The slight residual bias for small $S$ can be contrasted with results using Equation (7), which leads to a $95 \%$ downward bias in $\hat{N}_{\mathrm{e}}$ for $S=20$ and true $\hat{N}_{\mathrm{e}}=500$ (Figure 1).

\section{Monogamy}

For the monogamy model, $E\left(\hat{r}_{\text {sample }}^{2}\right)$ is the same as under random mating, and analyses similar to those described above led to the expressions for $E\left(\hat{r}_{\text {drift }}^{2}\right)$ and $\hat{N}_{\mathrm{e}}$ shown in Table 2 . Use of the revised $E\left(\hat{r}^{2}\right)$ with new simulated data resulted in estimates of $N_{\mathrm{e}}$ that performed at least as well as those for random mating (results not shown). Bias in $\hat{N}_{\mathrm{e}}$ was minimal for all parameter sets with large samples and for small samples when $S / N_{\mathrm{e}}>0.1$. Even with $S<30$ and $S / N_{\mathrm{e}}<0.1$, bias in $\hat{N}_{\mathrm{e}}$ was no more than $10 \%$.

\section{Precision}

With bias largely removed, it is important to at least briefly consider precision, because it can be a limiting factor for genetic estimates of effective size. A useful measure for this purpose is the quantity $\phi=V\left(\hat{r}^{2}\right) /\left(\hat{r}^{2}\right)^{2}=$ the square of the coefficient of variation of $\hat{r}^{2}$. Hill (1981) showed that if $\hat{r}^{2}$ is computed for a single pair of loci, $\phi \approx$ 2 , as would be expected if $\hat{r}^{2} / \hat{r}^{2}$ were a chi square variate with one degree of freedom. More generally, for $\hat{r}^{2}$ computed over all pairwise combinations of $L$ loci, if $J \hat{r}^{2} / \overline{\hat{r}}^{2}$ follows a chi square distribution with $J=L(L-1) / 2$ degrees of freedom, the following relationship should hold: $\phi \approx 2 / J ; J \phi$ $\approx 2$. That is, the variance of $\hat{r}^{2}$ should be inversely proportional to the number of pairwise comparisons of loci $(J)$ used in its computation.

Table 2. Parameters for estimating $N_{\mathrm{e}}$ for large and small sample sizes $(S)$ under two mating systems

\begin{tabular}{lll}
\hline & $S \geq 30$ & $S<30$ \\
\hline Random mating & $1 /\left(3 N_{\mathrm{e}}\right)-0.69 / N_{\mathrm{e}}^{2}$ & $308 / N_{\mathrm{e}}-0.52 / N_{\mathrm{e}}^{2}$ \\
$E\left(\hat{r}_{\text {drift }}^{2}\right)$ & $1 / S+3.19 / S^{2}$ & $0018+.907 / S+4.44 / S^{2}$ \\
$E\left(\hat{r}_{\text {sample }}^{2}\right)$ & $\frac{1 / 3+\sqrt{1 / 9-2.76 \hat{r}^{2 \prime}}}{2 \hat{r}^{2 \prime}}$ & $\frac{.308+\sqrt{.308^{2}-2.08 \hat{r}^{2 \prime}}}{2 \hat{r}^{2 \prime}}$ \\
$\hat{N}_{\mathrm{e}}$ & $2 /\left(3 N_{\mathrm{e}}\right)-1.8 / N_{\mathrm{e}}^{2}$ & $.618 / N_{\mathrm{e}}-1.31 / N_{\mathrm{e}}^{2}$ \\
Monogamy & $1 / S+3.19 / S^{2}$ & $.0018+.907 / S+4.44 / S^{2}$ \\
$E\left(\hat{r}_{\text {drift }}^{2}\right)$ & $\frac{2 / 3+\sqrt{4 / 9-7.2 \hat{r}^{2 \prime}}}{2 \hat{r}^{2 \prime}}$ & $\frac{.618+\sqrt{.618^{2}-5.24 \hat{r}^{2 \prime}}}{2 \hat{r}^{2 \prime}}$
\end{tabular}

$\hat{r}^{2 \prime}=\hat{r}^{2}-E\left(\hat{r}_{\text {sample }}^{2}\right)$ is the empirical $\hat{r}^{2}$ after subtracting the expected contribution from sampling for that model and sample size. 




Figure 4. The ratio $\hat{N}_{\mathrm{e}} / N_{\mathrm{e}}$ in the random mating model after bias correction $\left(\overline{\hat{r}}^{2}\right.$ values were used in modified expressions for $E\left(\hat{r}^{2}\right)$ shown in Table 2). (a) Large samples $(S \geq 30)$. (b) Small samples $(S=10-24)$. Results are based on new simulation data not used in deriving the bias corrections.

With $L=8$ loci, $J \phi$ is close to 2 provided that effective size is not too small (Figure 5a; random mating). However, when $N_{\mathrm{e}}<50, J \phi$ is higher than expected for a chi square variate, and it is much higher if $N_{\mathrm{e}}<25$ (Figure 5a). Comparable results were found for the monogamy model (Figure 5b).

To evaluate effects on hypothesis testing, I examined the frequency with which parametric confidence intervals (CIs) for $\hat{N}_{\mathrm{e}}$ based on point estimates of $\hat{r}^{2}$ contained the true $N_{\mathrm{e}}$. Confidence intervals were computed using an analogue of Equation (16) in Waples (1989):

$$
1-\alpha \mathrm{CI} \text { for } \hat{r}^{2}=\left[\frac{J\left(\hat{r}^{2}\right)}{\mathrm{X}_{\alpha / 2[]]}^{2}}, \frac{J\left(\hat{r}^{2}\right)}{\mathrm{X}_{1-\alpha / 2[]]}^{2}}\right],
$$

where $\mathrm{X}_{\alpha / 2[\lceil]}^{2}$ and $\mathrm{X}_{1-\alpha / 2[\eta]}^{2}$ are the $\alpha / 2$ and $1-\alpha / 2$ points of the chi square distribution with $J$ degrees of freedom. CIs for $\hat{r}^{2}$ were used with formulas in
Table 2 and Equation (7), respectively, to calculate CIs for $\hat{N}_{\mathrm{e}}$. I used $\alpha=0.05$ and the 0.025 and 0.975 points of the chi square distribution to calculate 95\% CIs for $\hat{N}_{\mathrm{e}}$.

Results of these analyses (Figure 6) show that a nearly linear (negative) relationship exists between the ratio $J \phi$ and the probability that the $95 \%$ CI for $\hat{N}_{\mathrm{e}}$ contains true $N_{\mathrm{e}}$. For $J \phi \leq 2$, the $95 \%$ CIs contained the true $N_{\mathrm{e}}$ at least $95 \%$ of the time, and performance dropped by about $5 \%$ for each unit of $J \phi$ above 2. Results for the monogamy model paralleled those of the random mating model (Figure 6).

Finally, I evaluated the effects on precision of using different numbers of loci to compute $\hat{r}^{2}$. In simulations using $N_{\mathrm{e}}=100, S=20,40$, or 100 and $L=2-20$ loci, $J \phi$ increased as a linear function of the number of loci (Figure 7). Because mean $\overline{\hat{r}}^{2}$ was essentially invariant for $L=2-20$ (data not shown), the increases were due to increases in the numerator $\left[J V\left(\hat{r}^{2}\right)\right]$ - that is, with increases in $J$ the variance of 


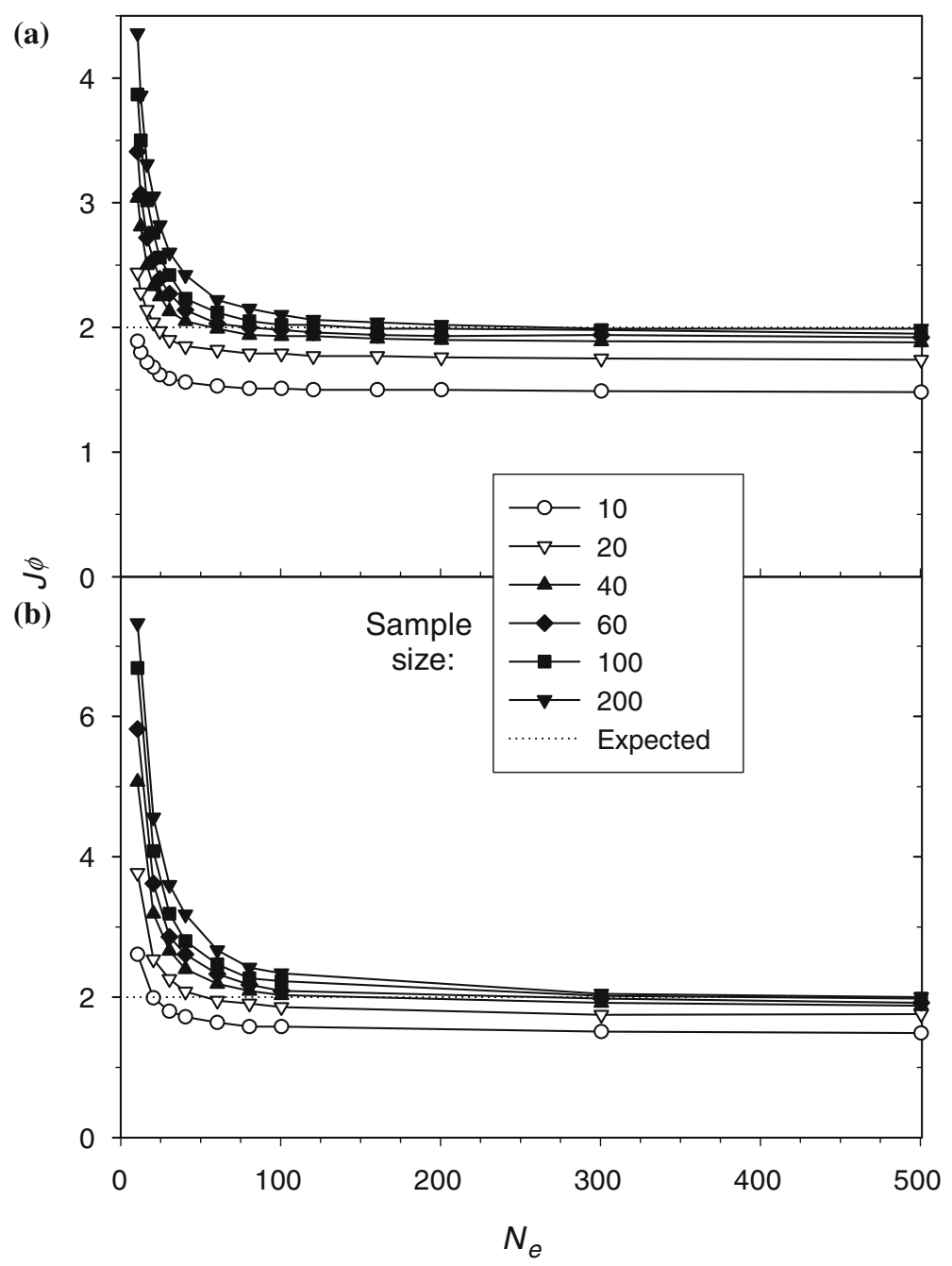

Figure 5. The ratio $J \phi$ for simulations with $L=8$ loci and a wide range of $S$ and $N_{\mathrm{e}}$ values. Dotted line represents expected value (2) assuming $J \hat{r}^{2} / \bar{r}^{2}$ is distributed as chi square with $J=L(L-1) / 2=28$ degrees of freedom. (a) Random mating model. (b) Permanent pair bonds model.

$\hat{r}^{2}$ did not decrease as rapidly as expected for variates following the chi square distribution. The effects on CIs of increases in $J \phi$ were similar to the pattern shown in Figure 7 for other simulations.

\section{Transforming $\hat{r}^{2}$}

I also considered an alternative bias correction method - to adjust empirical $\hat{r}^{2}$ and use the existing formula (Equation (7)) to calculate $\hat{N}_{\mathrm{e}}$. This is feasible because the relationship between $\overline{\hat{r}}^{2}$ and $E\left(\hat{r}^{2}\right)$ for any given sample size is almost perfectly linear (data not shown); therefore, for each sample size, a simple linear transformation of empirical $\overline{\hat{r}}^{2}$ values can bring them into agreement with $E\left(\hat{r}^{2}\right)$. Although this method proved to be as effective as the modified $E\left(\hat{r}^{2}\right)$ method in reducing bias in $\hat{N}_{\mathrm{e}}$, the linear transformation increased the variance of the adjusted $\overline{\hat{r}}^{2}$ and therefore reduced precision of $\hat{N}_{\mathrm{e}}$ (data not shown). Therefore, this latter method was not pursed further.

\section{Non-ideal populations}

The temporal method tracked $N_{\mathrm{e}}$ in non-ideal populations almost perfectly, both for skewed sex ratio (Figure 8a) and greater-than-random vari- 


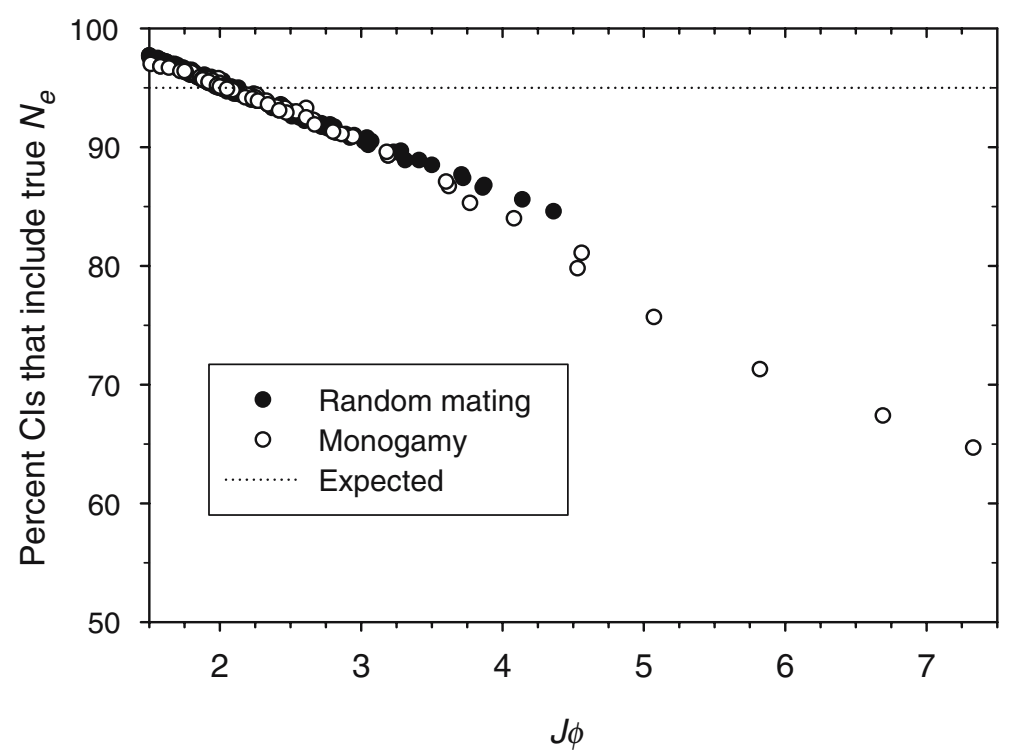

Figure 6. Performance of the confidence intervals (CIs) for $\hat{N}_{\mathrm{e}}$ as a function of $J \phi$. CIs for $\hat{N}_{\mathrm{e}}$ were based on CIs for $\hat{r}^{2}$ calculated using Equation (12). Filled symbols are for the random mating model, open circles for monogamy.

ance in reproductive success (Figure $8 b$ ). The modified linkage disequilibrium method also performed very well, although a consistent, slight upward bias in $\hat{N}_{\mathrm{e}}$ was evident (Figure 8). The cause of this slight bias is not clear, but it is inversely related to both $N_{\mathrm{e}}$ and $N_{\mathrm{e}} / N$. Upward bias in $\hat{N}_{\mathrm{e}}$ was less than $10 \%$ unless $N_{\mathrm{e}}^{2} / N$ was less than about 10 (data not shown).

\section{Discussion}

Bias

Sampling and drift effects

In spite of many years of concerted efforts, developing a theoretical expectation for $E\left(\hat{r}^{2}\right)$ has been difficult (Beaumont 2003). Several authors (e.g.,

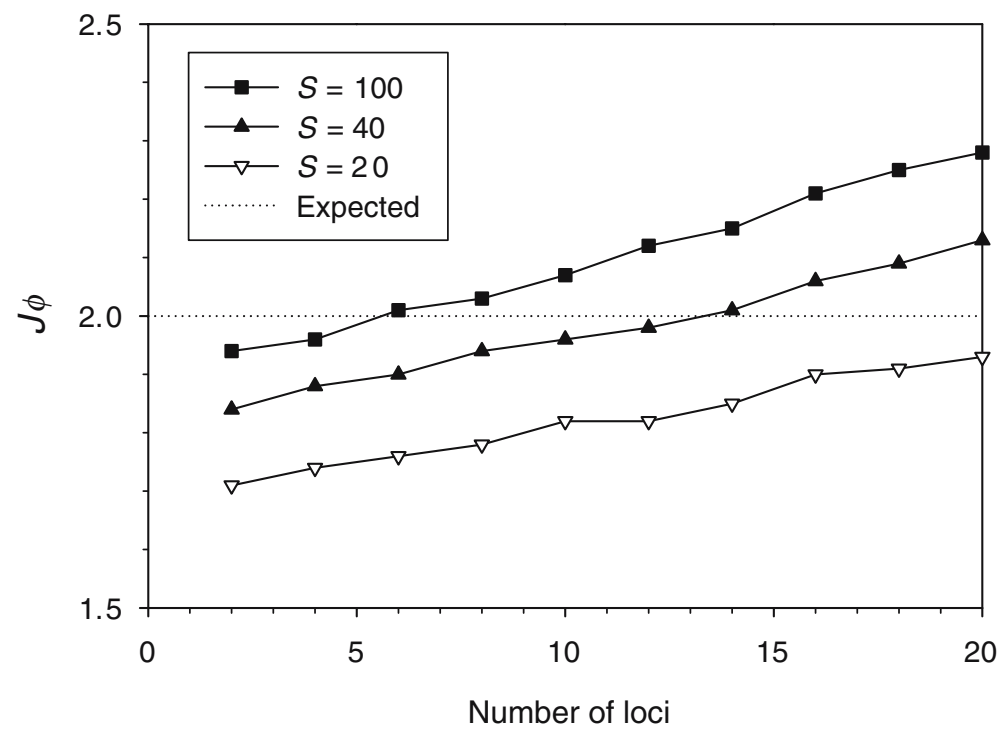

Figure 7. $J \phi$ for simulations using the random mating model with $N_{\mathrm{e}}=100$ and variable numbers $(L)$ of loci and sample sizes. Dotted line represents the value expected (2) assuming $J \hat{r}^{2} / \overline{\hat{r}}^{2}$ is distributed as chi square with $J=L(L-1) / 2$ degrees of freedom. 

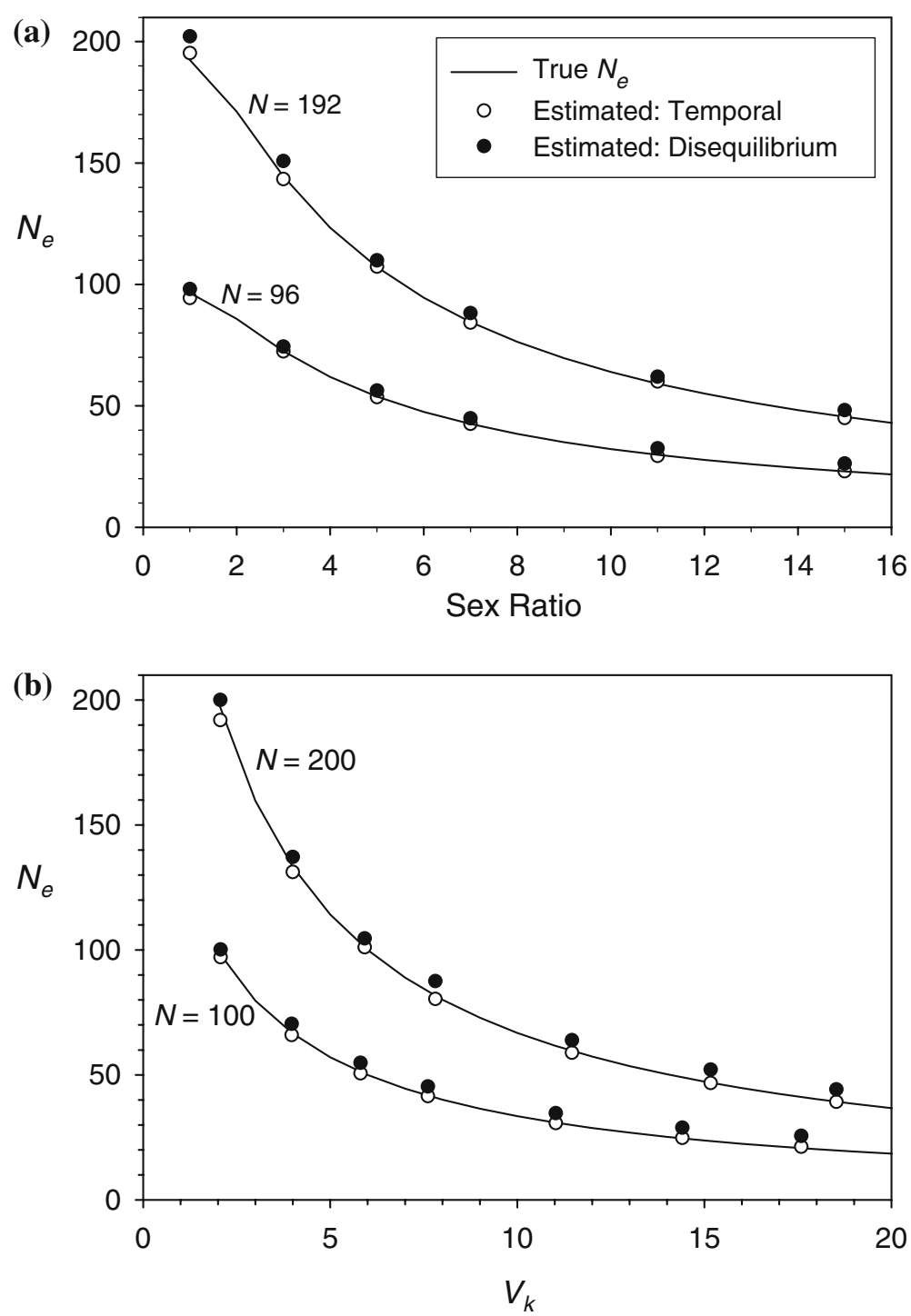

Figure 8. Comparison of estimated and true $N_{\mathrm{e}}$ in non-ideal populations. Open circles are estimates based on the temporal method; closed circles are estimates based on gametic disequilibrium using the modified expressions for $E\left(\hat{r}^{2}\right)$ given in Table 2. Populations were of fixed size $N$ as indicated and were ideal and random mating except for the following features: (a) Sex ratio ranged from 1:1 to 1:15. (b) Sex ratio equal but variance in reproductive success $\left(V_{\mathrm{k}}\right)$ ranged from Poisson $\left(V_{\mathrm{k}}=\bar{k}=2\right)$ to $V_{\mathrm{k}}=20$. Sample size was 50 and true $N_{\text {se }}$ was calculated using Equations (10) (Panel A) and (11) (Panel B).

Maruyama 1982; Golding 1984; Hudson 1985) have shown that the common approach of approximating $E\left(\hat{r}^{2}\right)$ as the ratio of two expectations can lead to bias. Weir and Hill (1980) provided the most rigorous derivation for $E\left(\hat{r}^{2}\right)$ but obtained their results only by assuming $S$ and $N_{\mathrm{e}}$ are both large. In real-world applications, however, sample sizes are generally limited and effective size is (or may be) small.
Inspection of Equations (5) and (6) shows that adequately accounting for sampling error is essential to obtain reliable estimates of $N_{\mathrm{e}}$. If $S$ and $N_{\mathrm{e}}$ are the same size, $E\left(\hat{r}_{\text {sample }}^{2}\right)$ is three times as large as $E\left(\hat{r}_{\mathrm{drift}}^{2}\right)$ in the random mating model and $50 \%$ higher in the monogamy model. Under these conditions, reliable estimates of $N_{\mathrm{e}}$ can be obtained only if two conditions are met: (1) the formula relating $E\left(\hat{r}^{2}\right)$ to $N_{\mathrm{e}}$ and $S$ is accurate; and 
(2) large amounts of data are available (sample sizes, number of loci), so that the actual contributions to $\hat{r}^{2}$ of drift and sampling are close to their expected values.

Results presented here confirm and expand the conclusion of England et al. (2006, this volume) that the first condition is not met with the most commonly used method for estimating effective population size based on gametic disequilibrium; rather, this method leads to substantial downward bias of $\hat{N}_{\mathrm{e}}$ if sample size is less than true $N_{\mathrm{e}}$. Figure 2, in which the effects of sampling are isolated from those due to drift, demonstrates that second-order terms that can safely be ignored when $S$ is large become important contributors to $\hat{r}^{2}$ when sample size is smaller. A similar result was found for $E\left(\hat{r}_{\text {drift }}^{2}\right)$ (Figure 3 ), and empirically derived second order terms in $S$ and $N_{\mathrm{e}}$ substantially improve the agreement between $\hat{r}^{2}$ and $E\left(\hat{r}^{2}\right)$. The small discrepancies that remain are probably due primarily to ignoring interaction terms in $S N_{\mathrm{e}}$.

Collectively, these results explain the pattern of bias seen in Figure 1. When $S / N_{\mathrm{e}}$ is small, sampling error affects $\hat{r}^{2}$ much more strongly that does drift, and $\hat{N}_{\mathrm{e}}$ is biased downwards because Equation (5) underestimates $E\left(\hat{r}_{\text {sample }}^{2}\right)$. Conversely, when $N_{\mathrm{e}}$ is small compared to $S$ (i.e., for large $\left.S / N_{\mathrm{e}}\right)$, the overestimation of $E\left(\hat{r}_{\mathrm{drift}}^{2}\right)$ is more important, leading to upward bias in $\hat{N}_{\mathrm{e}}$. In the random mating model for $S \geq 30$, these over- and under-estimation errors $\left(3.19 / S^{2}\right.$ and $-.69 / N_{\mathrm{e}}^{2}$; Table 2) cancel each other when $S=2.15 N_{\mathrm{e}}$, indicating that Equation (5) for $E\left(\hat{r}^{2}\right)$ should be unbiased only when $S / N_{\mathrm{e}} \sim 2.15$. That this is indeed the case is evident from Figure 1.

The modified equations for $E\left(\hat{r}^{2}\right)$ are effective enough that residual bias in $\hat{N}_{\mathrm{e}}$ (in most cases $<5 \%$ ) is not likely to lead to biologically misleading conclusions. After applying this correction, therefore, bias should be much less of a limitation for use of the disequilibrium method than is precision. Residual bias up to approximately $10 \%$ was found for $S<30$ and $S / N_{\mathrm{e}}<0.1$ (i.e., when $N_{\mathrm{e}}>300$ ).

England et al. (2006) suggested that situations in which $\hat{N}_{\mathrm{e}}$ is downwardly biased using the disequilibrium method might be identified by taking a series of subsamples of increasing size from the original sample. They reasoned that if the estimates of $\hat{N}_{\mathrm{e}}$ for the subsamples reach an asymp- totic value as size of the subsample increases, then $\hat{N}_{\mathrm{e}}$ for the complete data can be considered reliable, but if $\hat{N}_{\mathrm{e}}$ estimates continue to increase as size of the subsample increases, then true $N_{\mathrm{e}}$ is being underestimated by an unknown amount. Although this approach is reasonable in principle, it would not provide any redress for the latter case except to collect more individuals.

\section{Non-ideal populations}

Virtually all studies that have modeled the relationship between $N_{\mathrm{e}}$ and population genetic parameters have used ideal populations of different sizes. The implicit assumption is that an ideal population of $N_{\mathrm{e}}=N$ individuals is a reasonable proxy for all non-ideal populations of the same nominal effective size. Results presented in Figure 8 demonstrate that this is indeed a reasonable assumption for genetic estimators of effective size, regardless whether departures from ideal conditions are due to skewed sex ratio or overdispersed reproductive success. The slight upward bias in $\hat{N}_{\mathrm{e}}$ for the linkage disequilibrium method should not pose a significant problem in practical applications unless $N_{\mathrm{e}}$ and/or $N_{\mathrm{e}} / N$ are very small.

\section{Violation of model assumptions}

The models used here assume selective neutrality of unlinked markers and a single, closed population. Sensitivity of $\hat{r}^{2}$ and $\hat{N}_{\mathrm{e}}$ to violation of these assumptions remains to be studied in any systematic way. In structured populations, migration will lead to population mixture (and subsequent interbreeding will lead to admixture), which creates linkage disequilibrium due to a two-locus Wahlund effect (Sinnock 1975). Waples and Smouse (1990) considered the joint effects of population divergence, mixture fraction, and drift on linkage disequilibrium; although they did not estimate $N_{\mathrm{e}}$ directly, they showed that even with substantial population mixing, disequilibrium due to drift could dominate that due to mixture if population size was small. More recently, Vitalis and Couvet (2001) evaluated joint estimation of $N_{\mathrm{e}}$ and migration rate using both a single-locus measure and a two-locus measure related to $\hat{r}^{2}$. Several authors have examined power of linkage disequilibrium to detect selection, but potential biases that 
selection might create for estimating $N_{\mathrm{e}}$ have not been evaluated.

Although results presented here were not sensitive to allele frequencies in the range $0.05<P<0.95$, other studies (Maruyama 1982; Hudson 1985; Hedrick 1987; Waples and Smouse 1990) indicate that $\hat{r}^{2}$ is not independent of allele frequency. Bias in $\hat{N}_{\mathrm{e}}$ might be expected if allele frequencies are close to 0 or 1 but, again, a rigorous assessment remains to be done, particularly regarding the combined effects of many low-frequency alleles, such as might be encountered with data from microsatellites. Results presented by England et al. (2006 this volume) showing robustness of $\hat{N}_{\mathrm{e}}$ to varying numbers of alleles per locus are encouraging and suggest that challenges presented by loci with many alleles are not insurmountable for the disequilibrium method. Genotyping errors (Akey et al. 2001) are another source of potential bias for measures of gametic disequilibrium that have not been evaluated for their effects on $\hat{N}_{\mathrm{e}}$.

Formulas for $E\left(\hat{r}^{2}\right)$ presented here all apply to asymptotic values in populations of constant size. When $N$ varies, $\hat{r}^{2}$ will be affected by $N_{\mathrm{e}}$ in generations prior to the one sampled. Fortunately, for unlinked loci the approach to asymptotic $\hat{r}^{2}$ is rapid. Waples (2005) examined the specific time periods to which genetic estimates of $N_{\mathrm{e}}$ apply and concluded that recent population declines are not likely to seriously affect $\hat{N}_{\mathrm{e}}$ based on linkage disequilibrium, because the signal from current (reduced) $N_{\mathrm{e}}$ is strong relative to the signal from larger $N_{\mathrm{e}}$ in previous generations. This was also observed in the present study, where $\hat{N}_{\text {e }}$ quickly stabilized after initiating the simulations with $N_{\mathrm{e}}=\infty$. If $N$ has recently increased, $\hat{N}_{\mathrm{e}}$ can be biased downwards for a few generations, with duration and magnitude of bias proportional to severity of the bottleneck.

Linkage strongly affects $E\left(\hat{r}^{2}\right)$ and hence $\hat{N}_{\mathrm{e}}$ (Equations (3) and (4)). Linked loci provide greater precision in estimating $N_{\mathrm{e}}$ if $c$ is known (Hill 1981), but in that case $\hat{N}_{\mathrm{e}}$ will be more strongly affected by $N_{\mathrm{e}}$ in prior generations. Information for pairs of loci with different $c$ values can be combined as described by Hill (1981).

Results confirm the theoretical conclusion (Weir and Hill 1980) that different mating systems can have substantial effects on the expectation of $\hat{r}^{2}$. In particular, for species with separate sexes,
$E\left(\hat{r}^{2}\right)$ is much higher with lifetime monogamy than with completely random mating. Because Weir and Hill (1980) showed that $E\left(\hat{r}^{2}\right)$ for the random mating, separate sexes model is the same as $E\left(\hat{r}^{2}\right)$ for monoecious species with or without selfing, together the two mating systems considered here are applicable to a wide variety of organisms. As considerable bias could result from incorrect assumptions regarding lifetime monogamy, researchers should carefully consider this aspect of their study species. However, the encouraging results from modeling non-ideal populations suggest that lack of complete information about the mating system should not preclude general application of the disequilibrium method.

The assumption of discrete generations is violated by many (perhaps most) species; again, a rigorous treatment is lacking. In general, however, it can be noted that sampling a population with overlapping generations will provide an estimate of $N_{\mathrm{b}}$ (the effective number of breeders that produced the sample), rather than $N_{\mathrm{e}}$ (effective size for the generation). For example, if a single cohort is sampled, $\hat{N}_{\mathrm{b}}$ will provide information only about the effective number of breeders producing that cohort, not the population as a whole. The relationship between $N_{\mathrm{b}}$ and $N_{\mathrm{e}}$ can be complex (Waples 1991; 2002).

\section{Precision}

The sharp increase in $V\left(\hat{r}^{2}\right)$ for small $N_{\mathrm{e}}$ that is apparent in Figure 5 is probably an artifact of stochastic modeling of small populations. The expectation that $J \phi=2$ for a chi-square variate is valid only if $N_{\mathrm{e}}$ is constant. In the Wright-Fisher model used here, the expected variance in reproductive success was the binomial variance $=$ $\vec{k}(1-1 / N)$, but the actual $V_{\mathrm{k}}$ was a random variable that differed among replicates. As a consequence, actual $N_{\mathrm{e}}$ varied among replicates as well (cf Equation (11)). This inflates the variance in $\hat{r}^{2}$ and the effect is increasingly more pronounced as $N_{\mathrm{e}}$ gets smaller - the same pattern seen in Figure 5. Furthermore, this additional source of drift variance in $\hat{r}^{2}$ will be relatively more important when the contribution from sampling error is small, which explains why $J \phi$ is elevated more for larger sample sizes (Figure 5). It remains, however, to determine whether this phenomenon is sufficient to explain 
all of the elevated $V\left(\hat{r}^{2}\right)$ when $N_{\mathrm{e}}$ is small, or whether another factor is also involved.

$J \phi$ increases almost linearly with the number of loci (Figure 7) - the result that would be expected if $\hat{r}^{2}$ for individual locus pairs were not independent. Although the loci themselves are independent in the model used here, each pairwise comparison shares one locus with $L-2$ other pairwise comparisons. Hill (1981) did a limited evaluation of the correlation structure of $\hat{r}^{2}$ computed for different pairs of loci and found that correlations could be as high as $25 \%$ if one locus was shared in the two comparisons. It seems likely that results shown in Figure 7 reflect cumulative effects of relatively small dependencies among pairwise $\hat{r}^{2}$ values. In essence, $\hat{r}^{2}$ and $\hat{N}_{\mathrm{e}}$ behave as if they had fewer than $J$ degrees of freedom.

This topic merits further study. Analyses considered here only dealt with diallelic loci, whereas many genetic studies include data for highly polymorphic gene loci with many segregating alleles. A rigorous analysis of precision for the disequilibrium method would have to consider the effects of multiple alleles within loci (and their correlation structure) as well as effects of multiple loci. In the meantime, results in Figure 7 suggest that non-independence of pairwise comparisons is not likely to strongly affect precision of $\hat{N}_{\mathrm{e}}$. However, as precision can limit usefulness of the method, researchers should use as many individuals and loci as possible.

It is useful to compare precision of the linkage disequilibrium method with that of the more commonly used temporal method. As an example, assume one has two samples of $S=50$ individuals (using sampling Plan II; Waples 1989) and has obtained an estimate of $\hat{N}_{\mathrm{e}}=100$ for both the temporal method (using both samples) and the disequilibrium method (using either sample). How would confidence intervals for these estimates compare? This question can be answered for any given numbers of loci and alleles by computing confidence intervals for $\hat{r}^{2}$ (Equation (12)) and using the resulting values to compute CIs for $\hat{N}_{\mathrm{e}}$ (Table 2); analogous formulas (Waples 1989) accomplish the same thing for the temporal method. Figure 9 shows the range of CIs for $L=5$ or 20 loci, each having $a=2$ or 10 alleles. Each locus has the equivalent of $a-1$ independent alleles, and in the temporal method the number of degrees of freedom $(n)$ associated with $\hat{N}_{\mathrm{e}}$ increases as the product of

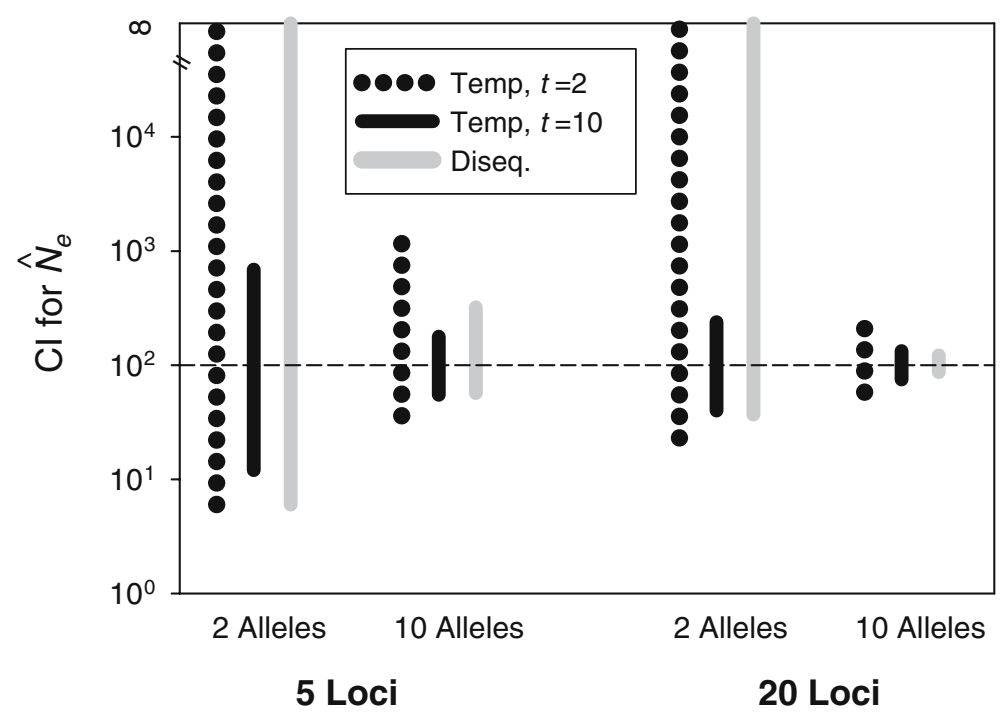

Figure 9. $95 \%$ confidence intervals (CIs) for $\hat{N}_{\mathrm{e}}$ in the temporal (Temp) and disequilibrium (Diseq) methods for different numbers of loci $(L)$, alleles per locus $(a)$, and number of generations $(t)$ between samples of 50 individuals each. These examples assume the point estimate $\hat{N}_{\mathrm{e}}$ is 100 , based on a pair of samples (temporal method) or a single sample (disequilibrium method). CIs for $\hat{r}^{2}$ were calculated using Equation (12) and resulting values were used to compute CIs for $\hat{N}_{\mathrm{e}}$ using the formula for random mating, $S>30$ in Table 2; Equations (16) and (11) in Waples (1989) were used in a similar way to generate CIs for the temporal method. Note that the exact time period(s) to which an estimate of $\hat{N}_{\mathrm{e}}$ applies is not necessarily the same for the two methods (see Waples 2005 for details). 
the numbers of loci and alleles $(n=L(a-1))$. In contrast, in the disequilibrium method the number of different pairwise comparisons of alleles at different loci (and hence the approximate degrees of freedom) increases as the square of this product $(n \approx L(L-1)(a-1)(a-1) / 2)$. In the temporal method, precision also increases with the number of generations between samples $(t)$. With only two alleles per locus (typical for allozymes or single-nucleotide polymorphisms), precision of both methods is poor except for the case of $t=10$ in the temporal method (Figure 9). However, with 10 alleles/locus, as commonly can be achieved with microsatellites, precision of the disequilibrium estimate exceeds that for a temporal estimate with $t=2$ and compares favorably with that of a temporal estimate based on 10 generations of drift. Results for the linkage disequilibrium method should be considered approximate, as data shown in Figure 7 and discussed above indicate that more work is needed to document the pattern of changes in $V\left(\hat{r}^{2}\right)$ for large numbers of loci and alleles. Nevertheless, it is apparent that under realistic scenarios for collecting genetic data for natural populations, the linkage disequilibrium method can provide estimates of $\hat{N}_{\mathrm{e}}$ with precision that rivals (and in some cases perhaps exceeds) that of the temporal method, and can do so with only a single sample of individuals.

\section{Acknowledgments}

I thank Gordon Luikart and Phillip England for bringing this bias to my attention and François Balloux for sharing an unpublished manuscript. Eric Anderson, Mark Beaumont, François Bonhomme, Craig Busack, Phillip England, Oscar Gaggiotti, Bill Hill, Gordon Luikart, Peter Smouse, David Tallmon, Bruce Weir, and two anonymous reviewers provided useful discussions and/or comments on earlier drafts. I am grateful to Geof Givens for suggesting the algorithm for generating an overdispersed Poisson distribution and to Craig Busack for pointing out the subtleties regarding the correction to $E\left(\hat{r}^{2}\right)$ for permanent pair bonding. A major part of this research was conducted while the author was a visiting scientist at LECA, and I am grateful to Gordon Luikart and Pierre Taberlet for making that possible.

\section{References}

Akey JM, Zhang K, Xiong M, Doris P, Jin L (2001) The effect that genotyping errors have on the robustness of common linkage disequlibrium measures. Am. J. Hum. Genet., 68, 1447-1456

Anderson EC (2001) Monte Carlo methods for inference in population genetic models, Ph.D. Dissertation, University of Washington, Seattle.

Ardren WR, Kapuscinski AR (2003) Demographic and genetic estimates of effective population size $\left(N_{\mathrm{e}}\right)$ reveals genetic compensation in steelhead trout. Mol. Ecol., 12, 35-49.

Bartley D, Bagley M, Gall G, Bentley M (1992) Use of linkage disequilibrium data to estimate effective size of hatchery and natural fish populations. Conserv. Biol., 6, 365-375.

Balloux F (2004) Heterozygote excess in small populations and the heterozygote-excess effective population size. Evolution, 58, 1891-1900

Beaumont MA (2003) Conservation genetics In: Handbook of Statistical Genetics (eds. Balding DJ, Bishop M, Cannings C) 2nd edn, pp. 751-792. Wiley, Chicester, UK.

Berthier P, Beaumont MA, Cornuet J-M, Luikart G (2002) Likelihood-based estimation of the effective population size using temporal changes in allele frequencies: a genealogical approach. Genetics, 160, 741-751.

Black CW, Krafsur ES (1985) A FORTRAN program for the calculation and analysis of two-locus linkage disequilibrium coefficients. Theoret. Appl. Genet., 70, 491-496.

Bucci G, Vendramin GG, Lelli L, Vicario F (1997) Assessing the genetic divergence of Pinus leucodermis Ant. endangered populations: use of molecular markers for conservation purposes. Theoret. Appl. Genet., 95, 1138-1146.

Caballero A (1994) Developments in the prediction of effective population size. Heredity, 73, 657-679.

Crow JF, Denniston C (1988) Inbreeding and variance effective population numbers. Evolution, 42, 482-495.

Crow JF, Morton NE (1995) Measurement of gene frequency drift in small populations. Evolution, 9, 202-214.

England PR, Cornuet J-M, Berthier P, Tallmon DA, Luikart G (2006) Estimating effective population size from linkage disequilibrium: severe bias using small samples. Cons. Genet. (this volume)

Frankham R (1995) Effective population size/adult population size ratios in wildlife: A review. Genet. Res., 66, 95-107.

Garnier-Gere P, Dillmann C (1992) A computer program for testing pairwise linkage disequilibria in subdivided populations. J. Heredity, 83, 239.

Golding GB (1984) The sampling distribution of linkage disequilibrium. Genetics, 108, 257-274.

Hauser L, Adcock GJ, Smith PJ, Ramírez JHB, Carvalho GR (2002) Loss of microsatellite diversity and low effective population size in an overexploited population of New Zealand snapper (Pagrus auratus). Proc. Nat. Acad. Sci. (US), 99, 11742-11747. 
Hedgecock D (1994) Does variance in reproductive success limit effective population sizes of marine organisms? In: Genetics and Evolution of Aquatic Organisms (ed. Beaumont AR), pp. 122-134. Chapman and Hall, London.

Hedrick PH (1987) Gametic disequilibrium: proceed with caution. Genetics, 117, 331-341.

Hill WG (1974) Estimation of linkage disequilibrium in randomly mating populations. Heredity, 33, 229-239.

Hill WG (1981) Estimation of effective population size from data on linkage disequilibrium. Genet. Res., 38, 209-216.

Hill WG, Robertson A (1968) Linkage disequilibrium in finite populations. Theoret. Appl. Genet., 38, 226-231.

Hudson RR (1985) The sampling distribution of linkage disequilibrium under an infinite allele model without selection. Genetics, 109, 611-631.

Hudson RR (2001) Linkage disequilibrium and recombination In: Handbook of Statistical Genetics (eds. Balding DJ, Bishop M, Cannings C), pp. 309-324. Wiley and Sons, New York.

Krimbas CB, Tsakas S (1971) The genetics of Dacus oleae. V. Changes of esterase polymorphism in a natural population following insecticide control - selection or drift? Evolution, 25, 454-460.

Laurie-Ahlberg C, Weir BS (1979) Allozyme variation and linkage disequilibrium in some laboratory populations of Drosophila melanogaster. Genet. Res., 32, 215-229.

Maruyama T (1982) Stochastic integrals and their application to population genetics In: Molecular Evolution, Protein Polymorphism, and the Neutral Theory (ed. Kimura M), pp. 151-166. Springer-Verlag, Berlin.

Nei M, Tajima F (1981) Genetic drift and estimation of effective population size. Genetics, 98, 625-640.

Nunney L (1993) The influence of mating system and overlapping generations on effective population size. Evolution, 47, 1329-1341.

Ohta T, Kimura M (1969) Linkage disequilibrium due to random genetic drift. Genet. Res., 13, 47-55.

Pollak E (1983) A new method for estimating the effective population size from allele frequency changes. Genetics, 104, 531-548.

Pritchard JK, Przeworski M (2001) Linkage disequilibrium in humans: Models and data. Am. J. Hum. Genet., 69, 1-14.

Raymond M, Rousset F (1995) GENEPOP (version 1.2): Population genetics software for exact tests and ecumenicism. J. Heredity, 86, 248-249.

Sinnock P (1975) The Wahlund effect for the two locus model. Amer. Nat., 109, 565-570.
Sved JA (1971) Linkage disequilibrium and homozygosity of chromosome segments in finite populations. Theoret. Pop. Biol., 2, 125-141.

Sved JA, Feldman MW (1973) Correlation and probability methods for one and two loci. Theoret. Pop. Biol., 4, 129-132.

Tallmon DA, Luikart G, Beaumont MA (2004) Comparative evaluation of a new effective population size estimator based on approximate Bayesian computation. Genetics, 167, 977-988.

Turner TF, Richardson LR, Gold JR (1999) Temporal genetic variation of mtDNA and effective female population size of red drum in the northern Gulf of Mexico. Mol. Ecol., 8, 1223-1230.

Vitalis R, Couvet D (2001) Estimation of effective population size and migration rate from one- and two-locus identity measures. Genetics, 157, 911-925.

Wang J (2001) A pseudo-likelihood method for estimating effective population size from temporally spaced samples. Genet. Res., 78, 243-257.

Waples RS (1989) A generalized approach for estimating effective population size from temporal changes in allele frequency. Genetics, 121, 379-391.

Waples RS (1991) Genetic methods for estimating the effective size of cetacean populations. Rep. Int. Whal. Commn. (special issue 13), 279-300.

Waples RS (2002) Definition and estimation of effective population size in the conservation of endangered species In: Population Viability Analysis (eds. Beissinger SR, McCullough DR), pp. 147-168. Univ. Chicago Press, Chicago.

Waples RS (2005) Genetic estimates of contemporary effective population size: To what time periods do the estimates apply? Mol. Ecol., 14, 3335-3352.

Waples RS, Smouse PE (1990) Gametic disequilibrium analysis as a means of identifying mixtures of salmon populations. Amer. Fish. Soc. Symp., 7, 439-458.

Weir BS (1979) Inferences about linkage disequilibrium. Biometrics, 35, 235-254.

Weir BS (1996) Genetic Data Analysis, 2nd edn. Sinauer, Sunderland, MA

Weir BS, Hill WG (1980) Effect of mating structure on variation in linkage disequilibrium. Genetics, 95, 447-488.

Williamson EG, Slatkin M (1999) Using maximum likelihood to estimate population size from temporal changes in allele frequencies. Genetics, 152, 755-761.

Wright S (1969) Evolution and the Genetics of Populations. Vol. 2. The Theory of Gene Frequencies, Univ. Chicago Press, Chicago. 